\title{
AVALIAÇÃO DO CURSO A DISTÂNCIA: PROMOÇÃO DA ALIMENTAÇÃO SAUDÁVEL NO AMBIENTE ESCOLAR, MINISTRADO PELO TELENUTRIÇÃO - RJ
}

\author{
Evaluation of the Impact of a Distance Course "Promoting Healthy Eating In School Environment" \\ Offered by Telenutrition - RJ
}

\author{
Maria Thereza Furtado Cury'; Joyce do Valle²; Tatiana Nóbrega³; Katheryn Ferreira de Assunção3; \\ Hadassa Gonçalves Di Lêu de Carvalho3 ${ }^{3}$ Priscila Rodrigues de Jesus ${ }^{3}$
}

\begin{abstract}
Resumo O Telessaúde-RJ mantém espaços específicos para cada profissão da área de saúde, tendo o projeto Telenutrição, como exemplo de um dos pioneiros desta iniciativa. Objetivos: Avaliar o desempenho do curso "Promoção da Alimentação Saudável no Ambiente Escolar". Metodologia: O levantamento de registros referente aos acessos dos usuários do Telenutrição no curso "Promoção da Alimentação Saudável no Ambiente Escolar" no período de 27 de Abril de 2012 a 4 de junho de 2012 foi obtido através dos dados gerados pelo programa Moodle. Os mesmos foram submetidos à análise estatística descritiva. Resultados: O curso obteve um total de 186 inscritos. Desses, 62,4 \% foram provenientes de cidades do interior. De todos os participantes, $43 \%$ concluíram o curso, sendo $65 \%$ usuários do interior e $35 \%$ das capitais. Os itens mais visualizados foram: aula, com 70,5\%, seguido por bibliografia, com $11,7 \%$. A região que obteve maior percentual de concluintes foi o Sudeste, representando $67,5 \%$. Conclusão: $O$ curso cumpriu seu objetivo, diante da adesão e conclusão de usuários de todas as regiões do Brasil, principalmente dos municípios externos às capitais.
\end{abstract}

Palavras-chave: Educação a Distância, Telessaúde, Cursos a Distância.

Abstract The Telehealth Center of State University of Rio de Janeiro (UERJ) involves different health professions, and Telenutrition is a pioneer example of this initiative. Aims: To evaluate the e-learning course "Promoting Healthy Eating in School Environments". Materials and methods: The access data in the e-learning course "Promoting Healthy Eating in School Environment" was obtained from the Telenutrition plataform covering the period from April 27, 2012 to June 4, 2012 by the Moodle program. The data were analyzed by descriptive statistical. Results: The course had a total of 186 registered persons. Of these, $62.4 \%$ came from the towns in the interior of different states of Brazil. Of all participants, $43 \%$ completed the course ( $65 \%$ from the towns in the interior). In the course, the most watched items were: lessons, with $70.5 \%$, followed by bibliography, with $11.7 \%$. Southeast was the region with the highest percentage of persons who have completed the course, representing $67.5 \%$. Conclusion: The e-learning course met its intended objective, evidenced by accession and completion by users from all regions of Brazil, especially from the towns in the interior.

Keywords: Distance Education, Telehealth, Telenutrition, Distance Learning Courses.

\footnotetext{
1. Professora Assistente do Instituto de Nutrição - Universidade do Estado do Rio de Janeiro. Doutoranda Programa de Pós-Graduação em Alimentação Nutrição e Saúde/ Instituto de Nutrição - Universidade do Estado do Rio de Janeiro; 2. Professora Associada do Instituto de Nutrição - Universidade do Estado do Rio de Janeiro; Professora Associada - Faculdade de Nutrição - Universidade Federal Fluminense; 3. Aluna do Curso de graduação em Nutrição do Instituto de Nutrição; estagiária do Telenutrição - Telessaúde - Universidade do Estado do Rio de Janeiro.
} 


\section{Introdução}

Atualmente, diversas mudanças tecnológicas estão afetando o modo de ver, viver, ser e de pensar do ser humano. $\mathrm{O}$ acelerado ritmo de vida das pessoas, as tecnologias incorporadas aos equipamentos de informática e de comunicação, a maior exigência de capacitação dos trabalhadores e o acirramento da concorrência no mercado de trabalho são fatores que têm levado muitas pessoas a buscarem meios de aperfeiçoamento e qualificação profissional através dos cursos mediados ou à distância ${ }^{1-3}$.

Assim, a prática da Educação a distância (EAD) transformou-se, no final do século $\mathrm{XX}$, numa das ferramentas utilizadas no âmbito da Educação. A Internet é um recurso que tem propiciado grande elasticidade à EAD ao permitir que a informação seja trocada de forma eficiente e veloz por um grande número de pessoas em diferentes lugares e ao mesmo tempo ${ }^{1,4}$.

Sendo assim, a EAD é vista como uma possibilidade viável na construção de mecanismos que favoreçam a aprendizagem e a qualificação contínuas ao longo da vida ${ }^{5}$

Nesse contexto, o Ministério da Saúde formalizou a rede de atualização e treinamento das Equipes de Saúde da Família (ESFs), por meio do TELESSAÚDE BRASIL. Trata-se de uma ação nacional que busca melhorar a qualidade do atendimento e da atenção básica no Sistema Único da Saúde (SUS), integrando ensino e serviço por meio de ferramentas de tecnologias da informação. O projeto, atualmente, é desenvolvido em doze dos vinte e seis estados brasileiros. No Estado do Rio de Janeiro (TELESSAÚDE-RJ), o programa é executado pela Universidade do Estado do Rio de Janeiro (UERJ) e tem como missão organizar, implantar e dar sustentabilidade a projetos em telemedicina e telessaúde nas áreas de pesquisa colaborativa, teleducação e teleassistência ${ }^{6,7}$.
Inserido no projeto está o TELENUTRIÇÃO, ferramenta de cunho preventivo, destacando a relação do ser humano com o alimento e mostrando sua importância fundamental na prevenção, manutenção e equilíbrio da saúde. É organizado e administrado como um projeto de extensão por uma equipe de professores do Instituto de Nutrição da UERJ.

Em relação aos cursos de atualização, os mesmos são realizados exclusivamente a distância e certificados pela Sub-reitoria de Extensão e Cultura da Universidade. Têm duração de 15 horas distribuídas entre as aulas e a avaliação escrita. Todos os nossos cursos têm arquivos com artigos relacionados a cada aula, disponíveis para leitura complementar. A participação do usuário se dá através da realização do login no site, onde são solicitados e-mail e senha. Em seguida, o interessado acessa o curso desejado e assiste aos módulos. Ao final, uma avaliação objetiva referente ao conteúdo abordado no curso é aplicada e uma nota mínima de $70 \%$ é necessária para emissão do certificado. É importante destacar que, além da nota mínima na prova, é preciso que o participante acesse todos os tópicos presentes no curso, além das aulas. Isso inclui o comunicado, a bibliografia e, mais recentemente, a avaliação do curso, que oportuniza a reflexão sobre o curso e sua prática e permite redimensionar seu planejamento, se necessário. Atualmente, contamos com três cursos oferecidos na área de nutrição, dois deles alocados na Rede de Atenção Primária: Promoção da Alimentação Saudável no Ambiente Escolar e Aconselhamento Nutricional da Obesidade na Infância e Adolescência e o último alocado na Rede Cegonha: Alimentação e Nutrição nas Doenças Associadas à Gestação. Este trabalho faz a avaliação e apresenta um dos cursos por nós oferecidos.

\section{Métodos}

O curso oferecido resulta da ação conjunta do Telenutrição com o NUCANE - Núcleo de Alimentação Escolar 
do INU/UERJ, que nesta parceria teve como objetivo articular ações institucionais que privilegiem processos de fortalecimento técnico junto aos nutricionistas responsáveis pelo Programa Nacional de Alimentação Escolar e outros profissionais interessados no tema.

Realizou-se levantamento dos registros dos relatórios gerados pelo ambiente de aprendizagem Moodle, no período 27 de Abril de 2012 a 4 de junho de 2012, referentes aos acessos ao curso "Promoção da Alimentação Saudável no Ambiente Escolar".

Foram utilizados os seguintes dados: acessos por atividade (ou seja, acessos ao curso em questão), acessos aos locais do sítio (aulas, bibliografias, prova, certificado e avaliação do curso), datas de acesso dos participantes/usuários, bem como Municípios e Estados dos profissionais e alunos inscritos. Os dados foram analisados usando estatística descritiva.

\section{Resultados e Discussão}

O curso obteve um total de 186 inscritos no período. Desses, 62,4\% eram oriundos das cidades do interior de todo o Brasil (Figura 1).

\begin{tabular}{|c|c|c|c|l|}
\hline Localização & Frequência & Percentual & $\begin{array}{c}\text { Percentual } \\
\text { Acumulado }\end{array}$ & \\
\hline Capital & 28 & $35,0 \%$ & $35,0 \%$ & $\square$ \\
\hline Interior & 52 & $65,0 \%$ & $100,0 \%$ & $\square$ \\
\hline Total & 80 & $100,0 \%$ & $100,0 \%$ & $\square$ \\
\hline
\end{tabular}

Figura 1: Percentual de concluintes por localização em cidades do interior e capital.

\begin{tabular}{|c|c|c|c|l|}
\hline Término & Frequência & Percentual & $\begin{array}{c}\text { Percentual } \\
\text { Acumulado }\end{array}$ & \\
\hline Não & 106 & $57,0 \%$ & $57,0 \%$ & $\square$ \\
\hline Sim & 80 & $43,0 \%$ & $100,0 \%$ & $\square$ \\
\hline Total & 186 & $100,0 \%$ & $100,0 \%$ & \\
\hline
\end{tabular}

Figura 2: Percentual de concluintes do curso em relação ao número de inscritos.
No Telessaúde do município Maravilha de Santa Catarina há uma média de 180 pessoas por palestra, número próximo dos 186 inscritos no curso analisado, segundo o próprio site do município com publicação da notícia em março de $2012^{8}$.

Cursos a distância e a utilização de ambientes virtuais de aprendizagem, buscam melhorar a qualidade dos serviços prestados em atenção primária, diminuindo o custo de saúde através da qualificação profissional. Este curso, uma demanda observada pelo NUCANE tinha como prioridade promover a formação permanente e a troca de experiências entre os profissionais envolvidos com a execução do PNAE, particularmente os responsáveis técnicos por esse programa nos 92 municípios do estado. Isto, talvez explique a proporção tão grande de usuários do interior, além das vantagens que a EAD apresenta, como menor custo, rompimento de barreiras pela distância, posto que o aluno não precisa se deslocar para ser treinado, liberdade no processo de aprendizagem com o cumprimento das tarefas, liberdade na ordenação temporal de aprendizagem, dentre outras.

Haguenauer (2003), ratificou que a educação a distância possibilita a flexibilização do tempo e espaço. Por este motivo há a adesão de alunos de todos os campos do país e estes apresentam chances de estudar a hora que quiserem. Há o ganho de tempo pelo não deslocamento para o local onde será realizado o curso, tempo pode ser revertido para o estudo. Este é um dos motivos da adesão de alunos de vários Estados no presente estudo. O autor observa que o acesso nas empresas é mais amplo quando os cursos são oferecidos por elas ${ }^{9}$.

De todos os participantes, $43 \%$ concluíram o curso, sendo $65 \%$ usuários do interior e $35 \%$ das capitais. (Figura 2).

Observamos que o percentual de não concluintes é alto, pois precisam de disciplina, motivação e equipamento bem dimensionado. Cerca de $6 \%$ e $10 \%$ usam internet discada e via radio, respectivamente. 


\begin{tabular}{|c|c|c|c|l|}
\hline Informação & Frequência & Percentual & $\begin{array}{c}\text { Percentual } \\
\text { Acumulado }\end{array}$ & \\
\hline Aula & 5440 & $70,5 \%$ & $70,5 \%$ & $\square$ \\
\hline $\begin{array}{c}\text { Avaliação do } \\
\text { Curso }\end{array}$ & 66 & $0,9 \%$ & $71,3 \%$ & $\square$ \\
\hline Bibliografia & 905 & $11,7 \%$ & $83,0 \%$ & $\square$ \\
\hline Certificado & 228 & $3,0 \%$ & $86,0 \%$ & $\square$ \\
\hline $\begin{array}{c}\text { Comunicado } \\
\text { Importante }\end{array}$ & 446 & $5,8 \%$ & $91,8 \%$ & $\square$ \\
\hline Prova & 635 & $8,2 \%$ & $100 \%$ & $\square$ \\
\hline Total & 7720 & $100 \%$ & $! 00 \%$ & $\square$ \\
\hline
\end{tabular}

Figura 3: Frequência de acessos aos locais do sítio. Itens mais visualizados.

Resultados obtidos por Araújo e Passos (2004) em uma análise no principal ambiente de educação a distância disponibilizado pela Escola do Futuro - USP apontaram 19 alunos matriculados; destes, 11 concluíram uma determinada disciplina eletiva oferecida. Este dado mostrou que a conclusão foi um pouco superior a $50 \%$, aproximando-se do resultado com $43 \%$ dos concluintes do curso analisado ${ }^{11}$.

Novaes et al. [s.d.] destacaram que a Rede NUTES (Rede de Núcleos do Telessaúde) de Pernambuco conseguiu obter o total de 369 participantes de agosto a dezembro no ano de 2003 e o curso analisado no período de abril até junho de 2012 obteve 186 inscritos. A Rede NUTES apresenta 27 sessões de videoconferências com várias áreas abordadas (clínica Médica, enfermagem, ginecologia e obstetrícia, pediatria e saúde mental), entretanto, vale lembrar que o curso analisado é apenas um dos oferecidos pelo Telenutrição do núcleo Telessaúde-RJ. Na Rede NUTES também há um questionário de avaliação para os participantes avaliarem as videoconferências, assim como no final do curso há um questionário para avaliação. Ao longo deste, disponibilizam-se consultas às bibliografias utilizadas para enriquecer o conhecimento do aluno ou sanar possíveis dúvidas que possam ter surgido no decorrer do curso ${ }^{6}$.
Os itens mais visualizados foram: AULA com 70,5\%, seguido por BIBLIOGRAFIA, com 11,7\% (Figura 3). A região que obteve maior percentual de concluintes foi a Sudeste, representando $67,5 \%$.

Nas visitas ao site, como era de se esperar, as aulas, num total de 10 , foram as mais visitadas, mas outra característica deste curso (a oferta de material de apoio às aulas para download) foi muito procurada. $\mathrm{Na}$ avaliação do curso, 95\% dos participantes dos cursos alegam que o curso atingiu suas expectativas. Os outros 5\% alegaram não terem gostado do curso; problemas técnicos com o som (quanto a volume e qualidade) em algumas aulas, bem como dificuldades no acesso do programa, relativas à velocidade da internet, foram fatos também bastante citados.

Observou-se também que a maior parte dos concluintes do curso reside no sudeste $(67,5 \%)$, onde estão localizados o estado do Rio de Janeiro e o Núcleo de Telessaúde-RJ. Este percentual também pode ser constatado pelo trabalho realizado pela equipe do Telenutrição, em 2010, que verificou que a região a obter maior percentual de visitas ao site foi a sudeste, com 82,9\% dos acessos. Desde este estudo, já se observa que a procura pelo site era realizada por visitantes fora do Estado do Rio de Janeiro e em todas as regiões brasileiras, ainda que de forma desigual. O tema com maior prevalência de acesso foi “nutrição e alimentação infantil", demonstrando que o curso analisado "Promoção da alimentação saudável no ambiente escolar" está inserido neste contexto ${ }^{7}$.

\section{Conclusão}

Nota-se que o projeto vem cumprindo seu objetivo por ser de extrema versatilidade. A implantação deste curso viabilizou o acesso para várias pessoas e há uma grande adesão por indivíduos de todas as regiões do Brasil. Os acessos de várias pessoas, tanto da capital 
quanto do interior, mostram que o curso teve uma difusão constante não se restringindo a apenas em uma parte ou a um Estado. O índice de conclusão do curso, de $43 \%$, é considerado bom, já que quase metade das pessoas que começaram o curso o concluiu, de fato.

Quando os profissionais têm acesso a cursos complementares e de atualização, toda a população passa a contar com equipes mais capacitadas e acesso a serviços especializados.

Apesar dos resultados positivos, há barreiras que devem ser rompidas quando se trata de educação a distância. Um desses exemplos é possibilitar o acesso regular a todos para esse tipo de serviço e estratégias de maior divulgação do espaço.

\section{Referências}

1-Pizzani L, Silva RC, Zornoff DCM, Arantes LF. A experiência pioneira do ensino à distância em treinamento de usuários de bibliotecas universitárias. RIED. 2012 ; Vol. 15 (1): $143-158$.

2-Almeida MEB. Educação a distância na internet: abordagens e contribuições dos ambientes digitais de aprendizagem. Educação e Pesquisa. 2003 Jul-Dez; Vol.29 (n.2): 327-340.

3-Spinardi ACP, Blasca WQ, Wen CL, Maximino LP. Telefonoaudiologia: ciência e tecnologia em saúde. Pró-Fono Revista de Atualização Científica. . 2009 jul-set; Vol.21(3):249-54

4-Todorov JC, Moreira MB, Martone RC. Sistema personalizado de ensino, educação à distância e aprendizagem centrada no aluno. Psicologia: Teoria e Pesquisa. 2009 Jul-Set; Vol. 25 (n. 3): 289-296.

5-Abbad GS, Zerbini T, Souza DBL. Panorama das pesquisas em educação a distância no Brasil. Estudos de Psicologia. 2010 Set-Dez; Vol.15( n. 3): 291-298.

6-Novaes MA, Araújo KS, Tavares SMB. Integração da universidade com a rede pública de saúde: experiência no desenvolvimento de um programa de videoconferência para capacitação em saúde. Núcleo de Telessaúde (NUTES). Universidade Federal de Pernambuco, Brasil [s.d.].

7- Cury MT, Valle J, Martins MA, Saturnino P. Telenutrição:educação permanente em nutrição para todos; 2010. In: World Nutrition Rio 2012. Rio de Janeiro; 2012.

8- Notícias do município Maravilha. Projeto para melhoria da saúde pública destaca trabalho realizado em Maravilha. Santa Catarina, 2012. http://www.maravilha. sc.gov.br/noticias/view/4646

9- Haguenauer C. Educação à distância e internet. LATEC - UFRJ. Rio de Janeiro; 2003

10 - Ghedine T, Testa MG, Freitas H. Educação à distância via internet em grandes empresas brasileiras. ERA. 2008: Out-Dez; Vol. 48 (n.4).

11- Araújo CA, Passos MSC. Otimizando comunidades de aprendizagem: análise de uma experiência de educação à distância. 2004: Abril. 